\title{
A declaração de óbito como indicador de sub-registro de casos de AIDS
}

\author{
Death certificates as a marker \\ for under-recording of AIDS cases
}

Katia Regina Valente de Lemos 1

Joaquim Gonçalves Valente 2

\footnotetext{
${ }^{1}$ Assessoria de DST/AIDS, Secretaria de Estado de Saúde do Rio de Janeiro. Rua México 128, sala 416, Rio de Janeiro, $R J$ 20031-142, Brasil. 2 Instituto de Medicina Social, Universidade do Estado do Rio de Janeiro. Rua São Francisco Xavier 524, 70 andar, Rio de Janeiro, $R J$ 20559-900, Brasil.
}

\begin{abstract}
This study aimed to estimate and evaluate the under-recording of AIDS cases that evolved to death. From 1991 to 1995, the Mortality Information System recorded 9,213 adult deaths due to AIDS, related by name, date of birth, and date of death to 15,505 AIDS recorded by the Disease Data Registration System from 1982 to 1996. This procedure showed that 51.9\% of AIDS deaths recorded in the period were not recorded as AIDS cases as they should have been. Univariate and multivariate analysis showed that women had a greater chance of under-recording than men $(O R=1.27)$. A lower probability of proper recording was observed in individuals with less schooling, and illiterates had a two-fold chance of not being properly recorded, as compared to individuals with college degrees. Deaths that occurred in health facilities classified as private units had a greater probability of not being recorded than those occurring elsewhere (mainly those classified as public reference units) $(O R=2.58)$. Deaths occurring in the city of Rio de Janeiro had a greater probability of under-recording than those in other cities $(O R=2.20)$. Key words Acquired Immunodeficiency Syndrome; Death Certificates; Underregistration
\end{abstract}

Resumo Este estudo teve como objetivo estimar e avaliar o sub-registro de casos de AIDS que evoluíram para óbito. Com base no nome e nas datas de nascimento e de óbito, foram relacionados 9.213 óbitos por AIDS, de indivíduos adultos, registrados pelo Sistema de Informação de Mortalidade (SIM) no período de 1991 a 1995, com 15.105 casos de AIDS registrados pelo Sistema de Informação de Agravos de Notificação (SINAN) no período de 1982 a 1996. Verificou-se que $51,9 \%$ dos óbitos não eram conhecidos do SINAN. As análises univariada e multivariada mostraram que as mulheres apresentaram maior chance de não terem sido notificadas do que os homens $(O R=1,27)$. Observou-se menor chance de notificação entre os indivíduos menos escolarizados, tendo os analfabetos chance duas vezes maior de não terem sido notificados que aqueles com nível universitário. Os óbitos ocorridos nas unidades de saúde privadas foram os que apresentaram maior chance de não terem sido notificados, principalmente se comparados com os óbitos ocorridos nas unidades classificadas como públicas de referência $(O R=2,58)$. Os óbitos ocorridos no Município do Rio de Janeiro apresentaram maior chance de não terem sido notificados que os ocorridos nos municipios do interior $(O R=2,20)$.

Palavras-chave Síndrome de Imunodeficiência Adquirida; Atestado de Óbito; Sub-registro 


\section{Introdução}

O sub-registro de casos de AIDS é um problema conhecidamente enfrentado pelo sistema de vigilância epidemiológica. Sabe-se que, em conseqüência de diversos fatores, dentre eles a subnotificação, o atraso nas notificações e no processamento das informações, os dados registrados sobre a ocorrência da doença não representam o número real de casos existentes. Estima-se que apenas um quinto dos casos de AIDS ocorridos no mundo são notificados à Organização Mundial de Saúde (OMS) (Mann et al., 1993). De acordo com dados da Joint United Nations Programme on HIVIAIDS (UNAIDS, 1996), estima-se que, nas Américas, excluindose os Estados Unidos, cerca de $60 \%$ dos casos de AIDS não são registrados

Embora seja inegável a importância da notificação dos casos de AIDS para o monitoramento da epidemia e para o conhecimento do comportamento da doença, declarações de óbito, listas de admissão hospitalar e registros de outras doenças são indicadas como fontes complementares de informação (Barchielli et al., 1995). Estudos têm sido realizados nos Estados Unidos e na Europa, com o objetivo de avaliar a qualidade e o grau de cobertura do sistema de vigilância epidemiológica e do sistema de mortalidade (Hardy et al., 1987; Johnson et al., 1989; Montellá-i-Jordana et al.,1995).

Um indicador importante na identificação de falhas no sistema de vigilância epidemiológica consiste na proporção de óbitos por AIDS não registrados anteriormente como casos de doença. Esse indicador tornou-se, no Brasil, ainda mais relevante a partir de 1996, quando o Ministério da Saúde (MS) introduziu o critério de confirmação de casos de AIDS a partir do óbito. Esse critério é exclusivo para adultos e determina que, após a investigação epidemiológica dos óbitos por AIDS e esgotadas todas as possibilidades de que o caso possa ser confirmado pelos critérios tradicionais, a declaração de óbito seja utilizada como critério de confirmação. Assim, os óbitos por AIDS são, a priori, casos confirmados da doença.

Nos Estados Unidos, um estudo que revisou atestados de óbito durante o período de três meses (julho a dezembro de 1985) em quatro cidades americanas (Hardy et al., 1987) verificou que, de 566 óbitos que tiveram como causa básica a AIDS, 414 (73\%) haviam sido registrados pelo sistema de vigilância. Dos 152 restantes, 98 (17\%) não tinham sido classificados como casos confirmados de AIDS e apenas 54 (10\%) eram realmente casos de AIDS não registrados.
Em Toscana, na Itália, o estudo realizado por Barchielli et al. (1995), no período de 1987 a 1991, comparou os casos de AIDS registrados pelo sistema de vigilância com os óbitos por AIDS registrados pelo sistema de mortalidade $\mathrm{e}$ concluiu pela alta cobertura do sistema de vigilância, com apenas cerca de 5,1\% dos óbitos por AIDS não tendo sido registrados como casos da doença.

Em estudo realizado em Ontário, Canadá, todos os óbitos por AIDS ocorridos no período de 1985 a 1987 foram relacionados com o registro local de casos de AIDS (Johnson et al., 1989). Os resultados mostraram que $24,8 \%$ dos óbitos por AIDS não constavam do registro de casos.

Entre os estudos nacionais, destaca-se o de Buchalla (1993), que revisou 4.023 declarações de óbitos de homens entre 20 e 49 anos de idade ocorridas no Município de São Paulo, no período de 1983 a 1986. Deste total, 359 foram identificadas como sendo de óbitos por AIDS, dos quais 54 (15\%) não estavam notificados como casos, embora em apenas 36 destes, a AIDS estivesse mencionada como causa básica ou associada.

De acordo com levantamentos realizados pelo Centro de Vigilância Epidemiológica da Secretaria de Estado da Saúde de São Paulo, entre 1991 e 1992, 15\% das notificações de AIDS chegaram ao conhecimento do sistema de vigilância por meio da declaração de óbito (Drumond Jr. et al., 1997). Um estudo mais recente realizado para o Estado de São Paulo (excluindo-se a capital paulista), mostrou que, de 3.170 óbitos que apresentavam a AIDS como causa básica, 971 (30,6\%) não eram conhecidos do sistema de vigilância (Barbieri et al.,1998).

No Estado do Rio de Janeiro, um estudo realizado pelo Programa de DST/AIDS da Secretaria de Estado de Saúde (SES/RJ) mostrou em seus resultados preliminares que, de um total de 1470 óbitos por AIDS ocorridos de 1993 a 1995, em 15 municípios do Estado, 53,3\% não constavam do banco de casos notificados da doença, indicando que grandes quantidades de casos têm sido conhecidas por meio da declaração de óbito (SES/RJ, 1997).

Vale ainda mencionar o trabalho de Ferreira \& Portela (1999), que, para avaliar a subnotificação dos casos de AIDS no Município do Rio de Janeiro no ano de 1996, relacionou as Autorizações de Internação Hospitalar (AIH) para AIDS com as notificações dos casos da doença. Os resultados mostraram uma subnotificação de $42,8 \%$.

Baseando-se no relacionamento dos casos de AIDS registrados pelo sistema de vigilância epidemiológica com os óbitos por AIDS regis- 
trados pelo sistema de mortalidade, o presente estudo teve como propósito a identificação e a análise dos óbitos por AIDS não registrados anteriormente como casos, objetivando não só estimar o sub-registro dos casos de AIDS que evoluíram para óbito, como também identificar possíveis fatores associados ao sub-registro. Buscou-se, desta forma, fornecer subsídios para o desenvolvimento de ações que visem à diminuição do sub-registro e ao aprimoramento do sistema de vigilância epidemiológica.

\section{Material e método}

\section{Fonte de dados}

Os óbitos foram obtidos a partir do Sistema de Informação de Mortalidade (SIM) e do Sistema Preliminar de Informação de Mortalidade (SPIM). O SPIM foi produzido pelo Departamento de Dados Vitais da Coordenadoria de Informação da SES/RJ, exclusivamente para as doenças que estão submetidas a algum tipo de vigilância epidemiológica. A necessidade de trabalhar com o SPIM foi determinada, na época da realização do estudo, pelo fato de haver nele duas informações não existentes no SIM: a variável nome, necessária para a avaliação do sub-registro, e a variável local de ocorrência. A codificação do óbito é referente à nona revisão da Classificação Internacional de Doenças.

As informações sobre os casos de AIDS foram obtidas a partir do Sistema de Informação de Agravos de Notificação (SINAN), em todo território nacional, a base de dados referentes às doenças de notificação.

\section{Critérios de inclusão}

Foram incluídos no estudo todos os óbitos ocorridos no Estado do Rio de Janeiro que tiveram a AIDS declarada como causa básica, no período de 1991 a 1995, entre indivíduos residentes no próprio Estado. A restrição quanto à residência e ocorrência dos óbitos deveu-se ao fato de não estarem ainda disponíveis os óbitos de residentes ocorridos em outras Unidades da Federação e à dificuldade em verificar se os óbitos de não residentes ocorridos no Estado do Rio de Janeiro haviam sido registrados em seus Estados de residência. Há que ressaltar, no entanto, que, no período estudado, menos de $1 \%$ das mortes por AIDS, entre indivíduos residentes no Estado do Rio de Janeiro, ocorreram em outras Unidades da Federação. Objetivando restringir o estudo aos óbitos que, $a$ prio$r i$, pudessem ser confirmados como casos de acordo com o critério "declaração de óbito", definido pelo MS e exclusivo para adultos, foram incluídos no estudo somente os óbitos de indivíduos com mais de 13 anos de idade, inclusive.

Os dados do SINAN utilizados neste estudo constituem-se de todos os casos de AIDS confirmados, de acordo com o critério de definição nacional (MS, 1992, 1994) em vigor na época (Critério CDC e OPAS/Caracas para adultos e CDC e Sinais Maiores e Menores para crianças), notificados ao Programa de Controle das DST/AIDS da SES/RJ até 31 de dezembro de 1996. Consideraram-se todos os casos de AIDS (adultos e crianças), porque indivíduos que morreram adultos podem ter sido registrados como casos ainda crianças (com menos de 13 anos).

\section{Tratamento dos dados}

O arquivo SIM foi relacionado ao arquivo SPIM a partir do número da declaração de óbito, uma variável comum aos dois arquivos. Foram selecionadas as variáveis data de nascimento, data do óbito, ano de ocorrência do óbito, sexo, idade, grau de instrução, estado civil, município de ocorrência (obtidas a partir do SIM), nome e local de ocorrência (obtidas a partir do SPIM).

Para diminuir os erros de registro, foram comparadas a idade registrada e a idade calculada a partir do ano de nascimento e do ano do óbito. Quando as idades diferiram em mais de dois anos, considerou-se a idade como ignorada.

Os óbitos foram agrupados em três grandes grupos etários (13 a 19 anos, 20 a 49 anos e 50 anos e mais).

Em virtude dos problemas operacionais resultantes de se trabalhar com diferentes unidades municipais em um mesmo período de tempo, optou-se por manter a distribuição geográfica de 1991. Assim, os óbitos ocorridos nos municípios emancipados a partir de 1992 foram incorporados aos seus municípios de origem. Em um primeiro momento, os municípios foram agrupados segundo as microrregiões geográficas definidas pela Fundação Instituto Brasileiro de Geografia e Estatística (IBGE). Posteriormente, pelo fato de $90 \%$ dos óbitos por AIDS ocorrerem na microrregião do Rio de Janeiro (constituída pelos municípios de Duque de Caxias, Itaboraí, Magé, Maricá, Nilópolis, Niterói, Nova Iguaçu, Rio de Janeiro, São Gonçalo e São João de Meriti) foram consideradas quatro áreas geográficas definidas como: $\mathrm{Mu}$ nicípio do Rio de Janeiro, Município de Niterói, outros municípios da microrregião do Rio de Janeiro e municípios do interior, que agrega os 
demais municípios do Estado. Os municípios do Rio de Janeiro e de Niterói foram considerados separadamente por apresentarem oferta de serviços de saúde diferenciada dos demais municípios da microrregião do Rio de Janeiro, bem como por responderem conjuntamente por $93 \%$ das ocorrências de óbitos por AIDS desta região.

A variável grau de instrução obedeceu à classificação disponível na declaração de óbito: nenhuma (analfabeto), fundamental (ensino fundamental completo ou incompleto), ensino médio (completo ou incompleto), universitário (completo ou incompleto) e ignorado. Esta variável foi considerada apenas para os indivíduos acima de 20 anos de idade.

Da mesma forma que para grau de instrução, a variável estado civil foi classificada de acordo com as informações disponíveis na declaração de óbito: solteiro, casado, viúvo, desquitado/divorciado, outros e ignorado e foi considerada apenas para os indivíduos acima de 20 anos.

O local de ocorrência do óbito foi considerado o lugar específico onde se deu o falecimento, podendo ser a unidade de saúde, o domicílio ou via pública. As unidades de saúde foram codificadas de acordo com o Relatório de Estabelecimentos Cadastrados por Município. Nos óbitos em que não houve informação e naqueles em que a informação disponível não permitiu identificar o local de ocorrência do óbito, esta variável foi codificada como ignorada. Entre as unidades de saúde, foram selecionadas aquelas com dez ocorrências de óbitos ou mais e agrupadas quanto à sua natureza privada (sem contrato com o SUS), privada conveniada (que mantém contrato com o SUS) e pública. As unidades públicas foram ainda classificadas de acordo com seu perfil assistencial em públicas de referência (hospitais que, além da tradição e volume no atendimento à AIDS, desenvolvem programas de treinamento de recursos humanos para a assistência à doença); públicas de emergência (hospitais considerados pelo Estado e municípios com perfil de emergência, além dos PAM - Postos de Assistência Médica); públicas especializadas (com perfil de atendimento direcionado a determinada especialidade médica) e públicas gerais (hospitais que não apresentam um perfil assistencial definido, incluindo aqueles em que o atendimento é exclusivo para determinada população, como, por exemplo, os hospitais militares ou o Hospital do IASERJ - Instituto de Assistência dos Servidores do Estado do Rio de Janeiro). As unidades de saúde com menos de dez ocorrências de óbito foram agrupadas em demais unidades de saúde, independente de sua natureza e perfil assistencial. Os óbitos ocorridos fora das unidades de saúde foram classificados como outros locais de ocorrência (óbitos domiciliares, em abrigos ou em via pública).

Para avaliar o sub-registro de casos de AIDS, foram relacionados o banco de dados do SIM, contendo os óbitos por AIDS de indivíduos adultos ocorridos no período de 1991 a 1995 já acrescidas as variáveis nome e local de ocorrência, e o banco de dados do SINAN, contendo todos os casos de AIDS notificados desde o início da epidemia em 1982, até 31 de dezembro de 1996 (15.105 casos). Para este procedimento, foram elaborados programas de computação em dBase III (Ashton-Tate, 1985), que, em um primeiro momento, fizeram a padronização da grafia de diversos nomes e, posteriormente, de acordo com os cinco critérios definidos por Lemos (1998), identificaram, a partir do nome, da data de nascimento e da data do óbito, os óbitos por AIDS registrados anteriormente como casos de doença.

Quando registrado no arquivo de casos (SINAN), o óbito foi considerado notificado; quando não registrado, foi considerado não notificado.

\section{Analise dos dados}

Foram calculados as proporções de óbitos não registrados no sistema de morbidade e os percentuais de casos conhecidos a partir da declaração do óbito, segundo ano de diagnóstico.

Foi verificada a associação entre a notificação e as variáveis ano de ocorrência do óbito, sexo, idade, área geográfica de ocorrência do óbito, local de ocorrência do óbito, grau de instrução e estado civil. Para tal, foram obtidas estimativas de odds ratios e seus respectivos intervalos de confiança. Foi realizada a análise multivariada, por meio da regressão logística, a fim de verificar o efeito de cada variável quando controladas todas as demais. O processo de modelagem estatística iniciou-se pelo modelo saturado, com posterior retirada das variáveis faixa etária e estado civil, que não apresentaram significância estatística e cuja não permanência no modelo não alterou de forma relevante os odds ratios das demais variáveis. A fim de que todos tivessem chance de ter cursado todos os graus de escolaridade, pelo fato de a idade não ter se mostrado significativamente associada à notificação e, principalmente, por haver apenas 68 óbitos de indivíduos entre 13 e 19 anos, foram incluídos no modelo final somente os indivíduos com 20 anos e mais de idade, inclusive. 


\section{Resultados}

Durante o período de 1991 a 1995, foram registrados pelo SIM 9.814 óbitos de indivíduos adultos que tiveram a AIDS como causa básica. Destes, 304 não foram encontrados no SPIM. Dos 9.510 óbitos restantes, foram retirados do estudo 110 em que houve incompatibilidade de informação entre o município de ocorrência do óbito e a instituição de ocorrência do mesmo, 19 em que não constava o nome, 61 que não apresentavam data de nascimento e 107 em que houve incompatibilidade entre a idade registrada e aquela calculada a partir das datas de óbito e nascimento. Desta forma, houve uma perda de 601 óbitos, o que corresponde a $6,1 \%$ do total de óbitos registrados oficialmente que cumpriam os critérios de inclusão no estudo.

Dos 9.213 óbitos restantes relacionados aos casos registrados pelo SINAN, $4.428(48,1 \%)$ eram conhecidos do registro de morbidade e 4.785 não o eram, o que corresponde a um subregistro de $51,9 \%$. A proporção de sub-registro para cada ano do estudo é apresentada na Figura 1.

Observa-se que o percentual de óbitos não registrados anteriormente como casos diminuiu ao longo dos anos e passou de $59,7 \%$, em 1991, para 45,2\%, em 1995 Essa tendência foi observada para ambos os sexos. Nos homens, o sub-registro passou de 59,4\%, em 1991, para $43,7 \%$, em 1995 , e, nas mulheres, de $61,6 \%$ para $50,9 \%$.

Levando-se em conta o número de casos de AIDS registrados no SINAN segundo o ano diagnóstico, foi possível, acrescidos os óbitos não registrados anteriormente como casos, corrigir o total de casos de AIDS para cada ano do estudo (Tabela 1).
Observa-se que, apenas para o período estudado, o número de casos confirmados de AIDS foi de 14.130, enquanto o total de casos acumulados desde o início da epidemia até dezembro de 1996, registrados pelo SINAN, foi de 15.105. A inclusão dos 4.785 casos de AIDS conhecidos por meio da declaração de óbito representaria um adicional de $51,2 \%$ no total de 9.345 casos diagnosticados entre 1991 e 1995.

A Tabela 2 mostra a proporção dos óbitos por AIDS não notificados anteriormente como casos, segundo cada variável selecionada.

Considerando-se as variáveis relacionadas a características individuais, observa-se que as

Figura 1

Proporção de óbitos por AIDS registrados como casos.

Estado do Rio de Janeiro, 1991 a 1995.

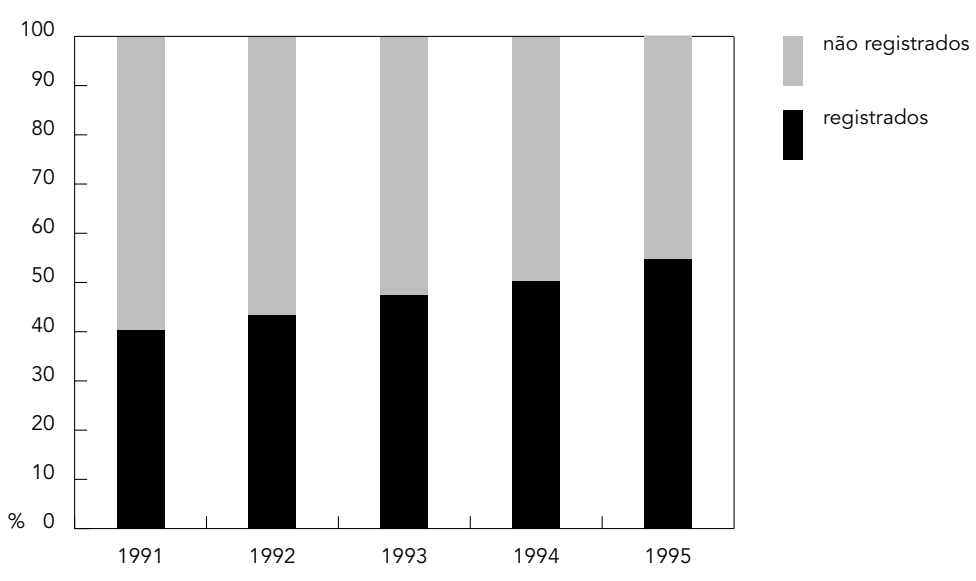

Tabela 1

Proporção de casos de AIDS conhecidos pela notificação do óbito segundo ano de diagnóstico.

Estado do Rio de Janeiro, 1991 a 1995.

\begin{tabular}{ccccc}
\hline Ano & Casos registrados $^{*}$ & $\begin{array}{l}\text { Óbitos por AIDS não } \\
\text { registrados como casos }\end{array}$ & $\begin{array}{c}\text { Total de casos } \\
\text { após correção }\end{array}$ & $\begin{array}{l}\text { Proporção de casos conhecidos } \\
\text { pela notificação do óbito }\end{array}$ \\
\hline 1991 & 1.618 & 844 & 2.462 & $34,3 \%$ \\
1992 & 1.937 & 898 & 2.835 & $31,7 \%$ \\
1993 & 1.990 & 964 & 2.954 & $32,6 \%$ \\
1994 & 1.967 & 1.067 & 3.034 & $35,2 \%$ \\
1995 & 1.833 & 1.012 & 2.845 & $35,6 \%$ \\
Total & 9.345 & 4.785 & 14.130 & $33,9 \%$ \\
\hline
\end{tabular}

* Casos registrados até 31/12/1996. 
Tabela 2

Freqüência absoluta e relativa dos óbitos por AIDS não notificados segundo variáveis selecionadas. Estado do Rio de Janeiro, 1991 a 1995.

\begin{tabular}{|c|c|c|c|}
\hline \multirow[t]{2}{*}{ Variáveis categóricas } & \multicolumn{2}{|c|}{ Não notificados } & \multirow[t]{2}{*}{ Total } \\
\hline & $\mathrm{n}$ & $\%$ & \\
\hline \multicolumn{4}{|l|}{ Sexo } \\
\hline Feminino & 1.002 & 56,07 & 1.787 \\
\hline Masculino & 3.777 & 50,95 & 7.413 \\
\hline \multicolumn{4}{|l|}{ Grupo etário } \\
\hline $13-19$ & 37 & 54,41 & 68 \\
\hline $20-40$ & 4.063 & 51,18 & 7.938 \\
\hline 50 e + & 685 & 56,75 & 1.207 \\
\hline \multicolumn{4}{|l|}{ Grau de instrução } \\
\hline Analfabeto & 177 & 60,41 & 293 \\
\hline Ensino fundamental & 2.175 & 54,61 & 3.983 \\
\hline Ensino médio & 927 & 49,15 & 1.886 \\
\hline Universitário & 474 & 45,19 & 1.049 \\
\hline \multicolumn{4}{|l|}{ Estado civil } \\
\hline Solteiro & 3.207 & 50,87 & 6.304 \\
\hline Casado & 996 & 53,52 & 1.861 \\
\hline Viúvo & 173 & 54,92 & 315 \\
\hline Divorciado & 144 & 54,96 & 262 \\
\hline Outros & 68 & 61,26 & 111 \\
\hline \multicolumn{4}{|l|}{ Local de ocorrência } \\
\hline Pública referência & 1.300 & 43,92 & 2.960 \\
\hline Pública emergência & 681 & 60,37 & 1.128 \\
\hline Pública especializada & 204 & 58,12 & 351 \\
\hline Pública geral & 854 & 54,26 & 1.574 \\
\hline Conveniada & 483 & 50,52 & 956 \\
\hline Privada & 289 & 58,15 & 497 \\
\hline Demais unidades & 399 & 65,84 & 606 \\
\hline Outros locais de ocorrência & 266 & 45,08 & 590 \\
\hline \multicolumn{4}{|l|}{ Área geográfica } \\
\hline Rio de Janeiro & 3.731 & 53,26 & 7.005 \\
\hline Niterói & 305 & 43,39 & 703 \\
\hline Outros municípios microrregião Rio & 360 & 66,18 & 544 \\
\hline Municípios do interior & 389 & 40,48 & 961 \\
\hline
\end{tabular}

maiores proporções de sub-registro ocorreram entre as mulheres, nos indivíduos acima de 50 anos, nos indivíduos menos escolarizados e nos não solteiros.

Levando-se em conta variáveis ligadas ao local de ocorrência do óbito, verifica-se que, excluindo-se as categorias demais unidades de saúde e outros locais de ocorrência, as maiores proporções de óbitos não notificados encontram-se entre aqueles ocorridos nos hospitais públicos de emergência. Quanto à área geográfica, observa-se que os óbitos ocorridos nos outros municípios da microrregião do Rio de Ja- neiro foram os que apresentaram maior proporção de sub-registro.

A Tabela 3 apresenta a odds ratio bruta e ajustada, segundo o modelo final da regressão logística.

Em conseqüência da falta de dados ( $m i s$ sing), 2.377 óbitos foram desconsiderados pelo modelo. Observou-se que o ano de morte apresenta-se positivamente associado com a notificação, tendo os indivíduos que faleceram em 1991 uma chance quase três vezes maior de não estarem notificados que aqueles falecidos em 1995.

Em relação ao sexo, observa-se que as mulheres apresentaram uma chance significativamente maior de não terem sido notificadas antes do seu falecimento do que os homens.

Os indivíduos entre 20 e 49 anos foram os que apresentaram menor chance de não terem sido notificados como caso antes de evoluírem para óbito. No entanto, não houve significância estatística.

Os indivíduos com nível universitário apresentaram menor chance de não notificação, diferindo de todos os outros grupos, principalmente do grupo de analfabetos, que tiveram uma chance quase duas vezes maior de não estarem notificados. Observou-se, ainda, que os óbitos de indivíduos com escolaridade em nível de ensino médio comparados àqueles de indivíduos de nível universitário apresentaram associação estatisticamente significativa com a notificação, o que não foi verificado na análise univariada.

Excluindo-se a categoria outros, as chances de os indivíduos solteiros não estarem notificados não diferiu de forma relevante nem estatisticamente significativa dos demais estados civis.

Quando consideradas determinadas categorias, o local de ocorrência do óbito mostrouse fortemente associado à não-notificação. Os indivíduos que morreram nos hospitais de referência tiveram menor chance de não terem sido registrados pelo sistema de morbidade antes de seu falecimento do que aqueles que morreram em qualquer outro grupo de hospitais. Essa chance chega a ser quase duas vezes menor quando considerados os hospitais de emergência.

Os óbitos ocorridos nos municípios classificados como do interior tiveram uma chance significativamente menor de não terem sido notificados como casos do que os ocorridos na capital e, principalmente, do que aqueles ocorridos nos outros municípios da microrregião do Rio de Janeiro. 
Odds ratio bruta e ajustada segundo modelo final da regressão logística não condicional, para não notificação. Estado do Rio de Janeiro, 1991 a 1995.

\begin{tabular}{|c|c|c|c|c|c|}
\hline Variáveis & OR bruta & IC $95 \%$ & OR ajustada & IC $95 \%$ & $p$ \\
\hline \multicolumn{6}{|l|}{ Ano } \\
\hline 1991/1995 & - & - & 2,71 & $2,27-3,23$ & $<0,0001$ \\
\hline $1992 / 1995$ & - & - & 1,99 & $1,70-2,32$ & $<0,0001$ \\
\hline 1993/1995 & - & - & 1,51 & $1,29-1,75$ & $<0,0001$ \\
\hline $1994 / 1995$ & - & - & 1,30 & $1,12-1,49$ & 0,0003 \\
\hline \multicolumn{6}{|l|}{ Sexo } \\
\hline Feminino/masculino & 1,23 & $1,11-1,37$ & 1,27 & $1,11-1,44$ & 0,0003 \\
\hline \multicolumn{6}{|l|}{ Grupo etário } \\
\hline $13-19 / 20-49$ & 1,14 & $0,69-1,84$ & - & - & - \\
\hline 50 e $+/ 20-49$ & 1,25 & $1,11-1,42$ & - & - & - \\
\hline \multicolumn{6}{|l|}{ Grau de instrução } \\
\hline Analfabeto/universitário & 1,85 & $1,41-2,43$ & 1,90 & $1,42-2,52$ & $<0,0001$ \\
\hline Fundamental/universitário & 1,46 & $1,27-1,68$ & 1,60 & $1,37-1,87$ & $<0,0001$ \\
\hline Médio/universitário & 1,17 & $1,00-1,37$ & 1,19 & $1,01-1,40$ & 0,0395 \\
\hline \multicolumn{6}{|l|}{ Estado civil } \\
\hline Casado/solteiro & 1,11 & $1,00-1,24$ & - & - & - \\
\hline Viúvo/solteiro & 1,18 & $0,93-1,49$ & - & - & - \\
\hline Divorciado/solteiro & 1,18 & $0,91-1,52$ & - & - & - \\
\hline Outros/solteiro & 1,53 & $1,02-2,28$ & - & - & - \\
\hline \multicolumn{6}{|l|}{ Local de ocorrência } \\
\hline Pub.emerg./pub. referência & 1,95 & $1,69-2,24$ & 1,96 & $1,67-2,30$ & $<0,0001$ \\
\hline Pub.especial./pub.referência & 1,77 & $1,41-2,23$ & 2,17 & $1,62-2,91$ & $<0,0001$ \\
\hline Pub.geral/pub.referência & 1,51 & $1,34-1,72$ & 2,35 & $2,01-2,74$ & $<0,0001$ \\
\hline Conveniada/pub. referência & 1,30 & $1,12-1,51$ & 1,95 & $1,63-2,32$ & $<0,0001$ \\
\hline Privada/pub. referência & 1,77 & $1,46-2,16$ & 2,58 & $2,06-3,22$ & $<0,0001$ \\
\hline Demais unidades/pub.ref. & 2,46 & $2,04-2,97$ & 3,61 & $2,84-4,59$ & $<0,0001$ \\
\hline Out.loc.ocorrência/pub ref. & 1,05 & $0,87-1,26$ & 1,27 & $1,03-1,55$ & 0,0248 \\
\hline \multicolumn{6}{|l|}{ Área geográfica } \\
\hline Rio/ municípios interior & 1,68 & $1,46-1,93$ & 2,20 & $1,75-2,77$ & $<0,0001$ \\
\hline Niterói/ municípios interior & 1,13 & $0,92-1,38$ & 1,35 & $1,01-1,80$ & 0,0044 \\
\hline $\begin{array}{l}\text { Outros munic. microrregião } \\
\text { Rio/ municípios interior }\end{array}$ & 2,88 & $2,30-3,61$ & 2,60 & $1,91-3,55$ & $<0,0001$ \\
\hline
\end{tabular}

\section{Discussão e conclusões}

Apresentam-se, como principais resultados desta análise, o alto percentual de sub-registro encontrado e as associações observadas entre a notificação e as variáveis sexo, grau de instrução, local de ocorrência, área geográfica de ocorrência e ano do óbito.

O fato de 51,9\% dos óbitos por AIDS não constar do registro de casos revela a baixa cobertura do sistema de vigilância epidemiológica no Estado, o que difere dos resultados obtidos em estudos internacionais que relaciona- ram registros de mortalidade com registros de casos. Em sua maioria, os estudos consultados concluíram pela alta cobertura do sistema de vigilância epidemiológica (Barchielli et al., 1996; Hardy et al., 1987; Montellá-i-Jordana et al., 1995), com o maior sub-registro encontrado $(24,6 \%)$ referindo-se a Ontario, Canadá (Johnson et al., 1989). Entre os trabalhos nacionais, o que melhor permite comparações com a presente análise é o estudo de Barbieri et al. (1998), o qual, examinando todos os óbitos por AIDS ocorridos em 1995 no Estado de São Paulo (excluindo-se a capital), verificou que $30,6 \%$ destes 
não eram conhecidos pelo sistema de vigilância. Assim, a comparação com estudos anteriormente realizados mostrou que a proporção de óbitos por AIDS não registrados como casos encontrada neste estudo foi superior àquelas observadas em estudos nacionais e internacionais.

Chama a atenção o aumento da cobertura do sistema de vigilância epidemiológica e conseqüente declínio do sub-registro. Além disto, os resultados da análise multivariada mostraram que os óbitos ocorridos em 1991 tiveram uma chance quase três vezes maior de não terem sido notificados que os ocorridos em 1995. A primeira hipótese aventada foi a de que, com a revisão nacional da definição do caso de AIDS em adultos, realizada em 1992 e tendo como um dos objetivos o aumento da sensibilidade da definição até então em vigor, elevou-se o número de casos confirmados de AIDS e, conseqüentemente, registrados, ocasionando, assim, a diminuição do sub-registro. Essa hipótese foi descartada por dois motivos: primeiro, pelo fato de que os casos anteriores a 1992 puderam ser confirmados de acordo com a nova definição e incorporados ao sistema; segundo, porque o decréscimo observado não foi marcado pelo ano de 1992, mas, ao contrário, caracterizou-se por uma diminuição contínua nos cinco anos observados. Resta, como alternativa, o aprimoramento gradual da qualidade do sistema de vigilância ao longo do período estudado.

O local de ocorrência do óbito mostrou-se fortemente relacionado à variável notificação e, como era esperado, os indivíduos que faleceram nas unidades de referência apresentaram menor chance de não estarem notificados em comparação com aqueles falecidos em todos os outros grupos de unidades de saúde. O único estudo encontrado que procurou identificar associações entre sub-registro e o local de ocorrência do óbito foi o de Ferreira \& Portela (1999), no qual as unidades de saúde foram agrupadas quanto à sua natureza jurídica, o que, pelo menos no momento, limita comparações com os resultados obtidos na presente análise.

Mesmo no modelo ajustado, a área geográfica de ocorrência do óbito manteve-se associada à não-notificação. $\mathrm{O}$ fato das menores chances de não-notificação terem sido observadas para os municípios do interior e de Niterói está provavelmente relacionado ao menor volume de atendimento, assim como a uma melhor organização dos serviços de saúde nessas localidades, o que propicia agilização na notificação e investigação dos casos de AIDS.

Em relação ao grau de instrução, observouse que as chances de não-notificação diminuem conforme se eleva o nível de escolarida- de. Resultados diferentes foram encontrados em estudos anteriores, como o de Buchalla (1993), que, analisando as mortes por AIDS e doenças associadas ocorridas entre homens de 20 a 49 anos no Município de São Paulo, no período de 1983 a 1986, identificou uma maior proporção de indivíduos com nível superior entre os óbitos não notificados como casos. Também no trabalho de Laumann (1989), em que os casos de AIDS identificados a partir de entrevistas realizadas para uma amostra nacional de domicílios nos Estados Unidos, no ano de 1988 , foram comparados com os dados de vigilância epidemiológica, foi observada uma maior subnotificação entre os casos identificados pelos indivíduos de estrato social mais alto. Ambos estudos, no entanto, referem-se a períodos da década de 80, quando dominava a ignorância e a impotência diante da doença, o que induziu muitos indivíduos, principalmente os de maior poder aquisitivo e geralmente atendidos por serviços de saúde privados, a solicitar ao médico a omissão do diagnóstico de AIDS, buscando dessa forma, livrarem-se de preconceitos e discriminações. Uma das hipóteses é a de que a crescente informação sobre a doença, a descoberta de um número cada vez maior de anti-retrovirais e, principalmente, a possibilidade de acesso a estes medicamentos por meio da rede pública de assistência tenham levado muitos indivíduos, particularmente aqueles com maior nível de informação, não só a terem uma postura favorável ao registro de seu verdadeiro diagnóstico, como a influenciar para que esse registro seja efetivado. Estes achados remetem ainda a um outro aspecto: sendo a chance de não-notificação maior entre os indivíduos com menor grau de instrução (um dos indicadores de classe social), as estimativas sobre as tendências de pauperização da epidemia, questão freqüentemente abordada no debate contemporâneo, podem, pelo menos quando os dados de registro são utilizados para subsidiar tais análises, estar subestimadas.

O sexo mostrou-se associado à notificação, não corroborando os achados dos estudos de Ferreira \& Portela (1999), para o Município do Rio de Janeiro, em 1996, e o de Johnson (1989), referente a Ontario, Canadá, no período de 1985 a 1987, onde não foram observadas associações estatisticamente significativas. $\mathrm{O}$ fato de as mulheres apresentarem maior chance de não estarem notificadas do que os homens pode advir do fato de, ainda hoje, estabeleceremse ligações imediatistas entre AIDS e os ditos grupos de risco (principalmente homossexuais masculinos), retardando o diagnóstico entre as mulheres. A associação entre sexo e notificação 
sinaliza para necessidade de integração das ações dos programas de controle de DST/AIDS e aqueles dirigidos à saúde da mulher.

Embora a idade tenha sido excluída do modelo final da análise multivariada, por não ter apresentado significância estatística, não deve ser totalmente descartada como um possível fator associado à subnotificação. O estudo de Ferreira \& Portela (1999), que inclui crianças em sua análise, observou maior subnotificação entre os menores de 13 anos.

O estado civil parece não ser realmente um fator importante na predição da notificação. Mesmo na análise univariada, as diferenças observadas não foram relevantes. Isto talvez se deva ao fato de o estado civil referir-se a uma situação jurídica e não a uma situação conjugal de fato. Assim, por exemplo, indivíduos com uma união consensual estável podem ter sido classificados como solteiros ou como outros, o que poderia estar atenuando as associações observadas.

Por fim, é importante acrescentar que este estudo tem, como principal limitação, as dificuldades decorrentes do relacionamento dos registros de óbitos (SIM) e casos (SINAN). Problemas como erros ortográficos e de digitação, falta de informações fundamentais, como é o caso da data de nascimento, dentre outros, foram obstáculos importantes que restringiram a utilização de programas de computação. Como conseqüência, é possível que casos registrados pelo sistema de vigilância tenham sido classificados como não notificados. Já os critérios uti- lizados para se considerar como sendo de um mesmo indivíduo os registros constantes do sistema de vigilância e de mortalidade foram suficientemente específicos, tornando bastante limitada a probabilidade de que indivíduos não registrados como casos no SINAN tenham sido considerados como notificados.

Este estudo teve como pressuposto que os óbitos apresentando como causa básica a AIDS constituíam-se em casos da doença. É possível, no entanto, que indivíduos soropositivos para o HIV, que apresentavam manifestações clínicas relacionadas à AIDS, porém que não cumpriam os critérios de definição de caso, tenham evoluído para óbito, sem que pudessem ser confirmados como casos de AIDS antes do seu falecimento, e, conseqüentemente, registrados pelo sistema de vigilância.

Concluiu-se que em $51,9 \%$ dos casos de AIDS que evoluíram para óbito, os indivíduos adoeceram e morreram sem terem sido registrados pelo sistema de vigilância epidemiológica. O fato de a subnotificação ter variado de acordo com o ano do óbito, o sexo, grau de instrução, local de ocorrência e área geográfica de ocorrência do óbito aponta para a necessidade de que esses fatores sejam considerados nas estratégias adotadas para a diminuição do subregistro e melhoria do sistema de notificação. O conhecimento do caso de AIDS por meio da declaração de óbito mostrou-se como um indicador relevante do sub-registro da doença, evidenciando a importância de se agregarem informações disponíveis em diferentes níveis.

\section{Referências}

ASHTON-TATE, 1985. Dbase III Plus Version 1.0 IBM/MSDOS. Washington, DC: Ashton-Tate.

BARBIERI, D.; SANTOS, N. I. S.; GUIBU, I. A.; JAMAL, L. F; CAMINADA, S. \& DOMINGUES C. S. B.,1998. Investigation of Cases and Deaths by AIDS from Death Certificates. In: 12th World AIDS Conference, Abstracts. CD-ROM. Geneva: Merck/Washington, DC: Whitehouse Station.

BARCHIELLI, A.; BUIATTI, E.; GALANTI, C.; GIOVANNETI, L.; ACCIAI, S. \& LAZZERI, V., 1995. Completeness of AIDS reporting and quality of AIDS death certification in Tuscany (Italy): A linkage study between surveillance system of cases and death certificates. European Journal of Epidemiology, 11:513-517. 
BUCHALLA, C. M., 1993. A Síndrome da Imunodeficiência Adquirida e a Mortalidade Masculina, de 20 a 49 Anos, no Município de São Paulo: 1983 a 1986. Tese de Doutorado, São Paulo: Faculdade de Saúde Pública, Universidade de São Paulo.

DRUMOND Jr., M.; LIRA, M. M. T. A.; FREITAS, M. \& NITRINI, T. M. V., 1997. A AIDS e os sistemas de informação de mortalidade em nível local: A experiência do PROAIM no Município se São Paulo. AIDS - Boletim Epidemiológico, 9:3-9.

FERREIRA, V. M. B. \& PORTELA, M. C. 1999. Avaliação da subnotificação de casos de AIDS no Município do Rio de Janeiro com base em dados do sistema de informação hospitalares do Sistema Único de Saúde. Cadernos de Saúde Pública, 15:317-324.

HARDY, A. M.; STARCHER, E. T.; MORGAN, W. M.; DRUKER, J.; KRISTAL, A.; DAY, J. M.; KELLY, C.; EWING, E. \& CURRAN, I. W., 1987. Review of death certificates to assess completeness of AIDS case reporting. Public Health Reports, 102:386-390.

JOHNSON, R. J.; MONTANO, B. L. \& WALLACE, E. M., 1989. Using death certificates to estimate the completeness of AIDS case reporting in Ontario in 1985-87. Canadian Medical Association Journal, 141:537-540.

LAUMANN, E. O.; GAGNON, J. H.; MICHAELS, S.; MICHAEL, R. T. \& COLEMAN J. S., 1989. Monitoring the AIDS epidemic in the United States: A network approach. Science, 244:1186-1189.

LEMOS, K. R. V., 1998. Mortalidade por AIDS no Estado do Rio de Janeiro e Avaliação do Subregistro de Casos de AIDS tendo como Indicador a Declaração de Óbito. Dissertação de Mestrado, Rio de Janeiro: Instituto de Medicina Social, Universidade do Estado do Rio de Janeiro.
MANN, J.; TARANTOLA, D. J. M. \& NETTER, T. W., 1993. AIDS no Mundo. Rio de Janeiro: Relume Dumará.

MONTELLÁ-i-JORDANA, N.; RICART-DE-MESONES, I.; BORRELL-i-THIÓ, C.; CLOS-i-GUIX, R. \& CAYLÀ-i-BURQUERAS, J., 1995. Comparación de las defunciones del registro de casos de sida y de las defunciones por sida del registro de mortalidad Barcelona; 1991-1992. Revista de Sanidade e Higiene Pública, 69:49-57.

MS (Ministério da Saúde), 1992. Revisão da Definição Nacional de Caso de AIDS em Adultos. Brasília: Programa Nacional de Controle de DST/AIDS, Ministério da Saúde.

MS (Ministério da Saúde), 1994. Revisão da Definição Nacional de Caso de AIDS em Crianças. Brasília: Programa Nacional de Controle de DST/AIDS, Ministério da Saúde.

SES/RJ (Secretaria de Estado de Saúde do Rio de Janeiro), 1997. Estudo de Sub-Registro de Casos e Óbitos de AIDS no Estado do Rio de Janeiro - Anos 1993,1994 e 1995: Segundo Relatório de Atividades. Rio de Janeiro: Divisão de Controle de DST/ AIDS, Secretaria de Estado de Saúde do Rio de Janeiro.

UNAIDS (Joint United Nations Programme on HIV/ AIDS), 1996. The HIVIAIDS Situation in Mid 1996: Global and Regional Highlights. Geneva: UNAIDS. 